\title{
Characterization and expression profiling of glutathione $S$-transferases in the diamondback moth, Plutella xylostella (L.)
}

Yanchun You ${ }^{1,2,3+}$, Miao Xie ${ }^{1,2,3+}$, Nana Ren ${ }^{1,3}$, Xuemin Cheng ${ }^{1,3}$, Jianyu Li ${ }^{1,2,3}$, Xiaoli Ma ${ }^{1,3}$, Minming Zou ${ }^{1,3}$, Liette Vasseur ${ }^{1,4}$, Geoff M Gurr ${ }^{1,3,5}$ and Minsheng You ${ }^{1,3^{*}}$

\begin{abstract}
Background: Glutathione S-transferases (GSTs) are multifunctional detoxification enzymes that play important roles in insects. The completion of several insect genome projects has enabled the identification and characterization of GST genes over recent years. This study presents a genome-wide investigation of the diamondback moth (DBM), Plutella xylostella, a species in which the GSTs are of special importance because this pest is highly resistant to many insecticides.

Results: A total of 22 putative cytosolic GSTs were identified from a published P. xylostella genome and grouped into 6 subclasses (with two unclassified). Delta, Epsilon and Omega GSTs were numerically superior with 5 genes for each of the subclasses. The resulting phylogenetic tree showed that the P. xylostella GSTs were all clustered into Lepidoptera-specific branches. Intron sites and phases as well as GSH binding sites were strongly conserved within each of the subclasses in the GSTs of P. xylostella. Transcriptome-, RNA-seq- and qRT-PCR-based analyses showed that the GST genes were developmental stage- and strain-specifically expressed. Most of the highly expressed genes in insecticide resistant strains were also predominantly expressed in the Malpighian tubules, midgut or epidermis.

Conclusions: To date, this is the most comprehensive study on genome-wide identification, characterization and expression profiling of the GST family in P. xylostella. The diversified features and expression patterns of the GSTs are inferred to be associated with the capacity of this species to develop resistance to a wide range of pesticides and biological toxins. Our findings provide a base for functional research on specific GST genes, a better understanding of the evolution of insecticide resistance, and strategies for more sustainable management of the pest.
\end{abstract}

Keywords: Transcriptome analysis, qRT-PCR, Phylogenetic analysis, Insect pest, Lepidoptera

\section{Background}

The diamondback moth (DBM), Plutella xylostella (L.) (Lepidoptera: Plutellidae), is a world-wide destructive pest of wild and cultivated crucifers [1]. The larvae feed on cruciferous plants and may cause significant reductions in yield and quality of economically important crops such as canola and cabbage. Historical reliance on insecticides has led to the rapid development of

\footnotetext{
* Correspondence: msyou@iae.fjau.edu.cn

${ }^{\dagger}$ Equal contributors

'Institute of Applied Ecology and Research Centre for Biodiversity and Eco-Safety, Fujian Agriculture and Forestry University, Fuzhou 350002, China ${ }^{3}$ Key Laboratory of Integrated Pest Management of Fujian and Taiwan, China Ministry of Agriculture, Fuzhou 350002, China

Full list of author information is available at the end of the article
}

resistance in $P$. xylostella populations [2], making it difficult to control.

Several studies have examined the potential mechanisms underlying the development of insecticide resistance in P. xylostella [3-5]. One of the proposed mechanisms is metabolic resistance through the multifunctional glutathione $S$-transferases (GSTs, EC2.5.1.18). These enzymes can catalyze electrophilic compounds, making them water soluble and readily excreted [6]. GSTs are known more generally by insects to detoxify various xenobiotics, including insecticides and plant allelochemicals [7]. The recent work has focused on the potential role of GSTs in oxidative stress responses [6,8-11].

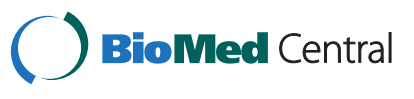

(c) 2015 You et al.; licensee BioMed Central. This is an Open Access article distributed under the terms of the Creative Commons Attribution License (http://creativecommons.org/licenses/by/4.0), which permits unrestricted use, distribution, and reproduction in any medium, provided the original work is properly credited. The Creative Commons Public Domain Dedication waiver (http://creativecommons.org/publicdomain/zero/1.0/) applies to the data made available in this article, unless otherwise stated. 
Table 1 Description of 22 identified cytosolic GSTs in the $P$. xylostella genome

\begin{tabular}{|c|c|c|c|c|c|}
\hline $\begin{array}{l}\text { Gene } \\
\text { name }\end{array}$ & $\begin{array}{l}\text { ORF } \\
\text { (bp) }\end{array}$ & $\begin{array}{l}\text { Protein } \\
\text { (AA) }\end{array}$ & $\begin{array}{l}\text { Gene size } \\
\text { (bp) }\end{array}$ & $\begin{array}{l}\text { Scaffold/ } \\
\text { orientation }\end{array}$ & Gene ID ${ }^{a}$ \\
\hline$\overline{P x G S T d 1^{b}}$ & 654 & 217 & 2534 & $38 /+$ & Px010343 \\
\hline PxGSTd2 & 648 & 215 & 3031 & $75 /+$ & Px015896 \\
\hline PXGSTd3 ${ }^{b}$ & 660 & 219 & 1640 & $75 /-$ & Px015897 \\
\hline PxGSTd4 & 672 & 223 & 2321 & $221 /+$ & Px006286 \\
\hline PxGSTd5 & 672 & 223 & 2294 & 73/- & Px015631 \\
\hline PxGSTe1 & 699 & 232 & 1823 & $66 /+$ & Px014816 \\
\hline PxGSTe2 ${ }^{\mathrm{b}}$ & 684 & 227 & 3497 & $363 /-$ & Px010078 \\
\hline PxGSTe3 & 687 & 228 & 1699 & $41 /+$ & Px011036 \\
\hline PxGSTe4 & 663 & 220 & 2922 & $216 /+$ & Px006106 \\
\hline PxGSTe5 & 651 & 216 & 4863 & $216 /+$ & Px006105 \\
\hline PxGSTO1 & 768 & 255 & 2649 & $85 /+$ & Px016897 \\
\hline PxGSTO2 ${ }^{\mathrm{b}}$ & 750 & 249 & 6717 & $554 /+$ & Px016898 \\
\hline PxGSTO3 & 726 & 241 & 3721 & $25 /+$ & Px007118 \\
\hline PxGSTO4 & 750 & 249 & 750 & $7 /-$ & Px015266 \\
\hline PxGSTO5 & 738 & 245 & 2200 & $554 /+$ & Px013473 \\
\hline PXGSTS1 & 615 & 204 & 2669 & $320 /-$ & Px009113 \\
\hline PxGSTs2 & 615 & 204 & 8124 & $328 /-$ & Px009257 \\
\hline PXGSTt1 & 654 & 217 & 3261 & $547 /-$ & Px000759 \\
\hline PxGSTu1 & 693 & 230 & 2771 & 1088/- & Px000790 \\
\hline PXGSTU2 & 648 & 215 & 963 & 408/- & Px010993 \\
\hline PxGSTZ1 & 645 & 214 & 2450 & $16 /-$ & $P \times 003659$ \\
\hline PxGSTz2 & 642 & 213 & 15681 & $115 /+$ & Px001225 \\
\hline
\end{tabular}

${ }^{a}$ The gene IDs were obtained directly from the published DBM genomic database (DBM-DB: http://iae.fafu.edu.cn/DBM/family/PxGSTs.php). All the coding sequences (CDS) of the PxGST genes have been experimentally validated. ${ }^{b}$ Coding sequences of such genes were incomplete from the DBM-DB, and have been experimentally completed by PCR, as explained in the methodology.

Px: Plutella xylostella.
Insect GSTs are classified as cytosolic and microsomal. The number of microsomal GSTs is much lower than that of cytosolic GSTs, which have been grouped into six subclasses [12]. Delta and Epsilon subclasses are insect specific, while the other four subclasses, Omega, Sigma, Theta, and Zeta, are found in various animal taxa $[10,13,14]$.

GSTs are involved in the resistance of insects to organophosphate (OPs), chlorine, and pyrethroid insecticides [15,16]. Recombinant GST enzymes from $P$. xylostella and Drosophila melanogaster have been shown to play a role in the metabolism of organophosphate insecticides $[17,18]$. It has been suggested that, under elevated GST activity conditions, Anopheles subpictus can detoxify fenitrooxon activation products, leading to organophosphate resistance [19]. The silkworm Zeta GST recombinant protein (rbmGSTz) has been found to initiate the dechlorination of permethrin and to be abundantly distributed in a permethrin-resistant strain [20]. Similarly, an Omega GST is highly expressed in a fenitrothion-resistant strain of silkworm and its recombinant protein (rbmGSTo) shows high affinity with organophosphate insecticides, indicating that it may contribute to insecticide resistance and oxidative stress responses [21]. The antennae-specific GST was found being involved with detoxification of xenobiotics and detection of sex pheromones in Manduca sexta [22].

The GSTs were found to be one of the major enzyme families in the P. xylostella genome and to be linked to detoxification of plant defense compounds and insecticides [23]. A recent study on the identification and characterization of multiple glutathione $S$-transferase genes [24] based on the DBM transcriptome database $[24,25]$ provides a primary base for further investigation of this important gene family. In the present study, the P. xylostella GSTs (PxGSTs) were identified and compared with the equivalent information from published

Table 2 Comparison of GST gene numbers of various insect species*

\begin{tabular}{|c|c|c|c|c|c|c|c|c|}
\hline Insect species & Delta & Epsilon & Omega & Sigma & Theta & Zeta & Unclassified & Total \\
\hline P. xylostella & 5 & 5 & 5 & 2 & 1 & 2 & 2 & 22 \\
\hline B. mori & 4 & 8 & 4 & 2 & 1 & 2 & 2 & 23 \\
\hline C. quinquefasciatus & 17 & 10 & 1 & 2 & 6 & 0 & 3 & 39 \\
\hline D. melanogaster & 11 & 14 & 5 & 1 & 4 & 2 & 0 & 37 \\
\hline A. gambiae & 12 & 8 & 1 & 1 & 2 & 1 & 3 & 28 \\
\hline A. aegypti & 8 & 8 & 1 & 1 & 4 & 1 & 3 & 27 \\
\hline T. castaneum & 3 & 19 & 3 & 7 & 1 & 1 & 2 & 36 \\
\hline N. vitripennis & 5 & 0 & 2 & 8 & 3 & 1 & 0 & 19 \\
\hline A. mellifera & 1 & 0 & 1 & 4 & 1 & 1 & 0 & 8 \\
\hline A. pisum & 9 & 0 & 2 & 5 & 2 & 0 & 6 & 24 \\
\hline P. humanus & 4 & 0 & 1 & 4 & 1 & 1 & 0 & 11 \\
\hline
\end{tabular}

*Data were from cited literature: Friedman (2011) [30], Oakeshott et al. (2010) [31], Yu et al. (2008) [28], Ding et al.(2003) [29] and Nair et al.(2011) [32]. 


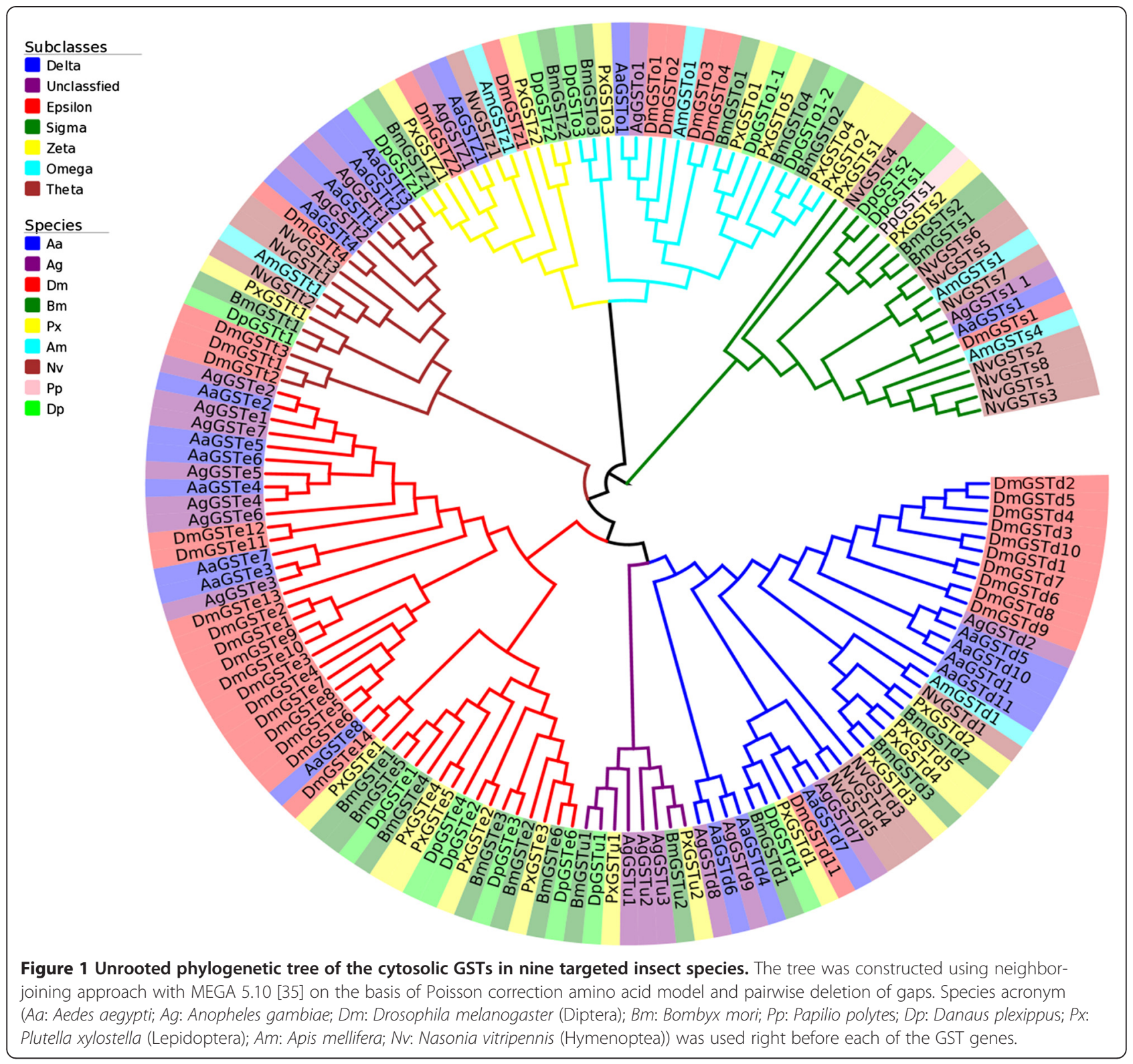

insect genomes to better reveal their phylogenetic relationships and intron-exon organization. We profiled and analyzed expression patterns of the PxGSTs using the published transcriptome [26] and reverse transcriptionquantitative polymerase chain reaction (qRT-PCR) in different life stages and tissues from insecticide susceptible or resistant strains. We then examined the major characteristics of GST subclasses and some particular GST genes in relation to their potential roles in P. xylostella insecticide resistance.

\section{Results and discussion}

Identification of the PxGSTs

Queries for PxGSTs were done against the amino acid sequences from the other insects: Drosophila melanogaster
(Dm), Culex quinquefasciatus (Cq), Aedes aegypti (Aa), Anopheles gambiae (Ag) (Diptera), Tribolium castaneum (Tc) (Coleoptera), Apis mellifera (Am), Nasonia vitripennis $(\mathrm{Nv})$ (Hymenoptea), Pediculus humanus (Ph), Acyrthosiphon pisum (Ap) (Exopterogota), and Bombyx mori (Bm) (Lepidoptera). Twenty-two putative cytosolic GST genes with full-length sequence were identified from our $P$. xylostella $(P x)$ genome [23,27] (Table 1) and further validated by cloning and sequencing. Using the listed gene IDs in Table 1, the coding sequences (Additional file 1), inferred amino acid sequences (Additional file 2) and genomic DNA sequences (Additional file 3) can be found in the published DBM genomic database (DBM-DB: http:// iae.fafu.edu.cn/DBM/family/PxGSTs.php) [27]. Compared to the previous DBM GSTs [24] identified from a published 


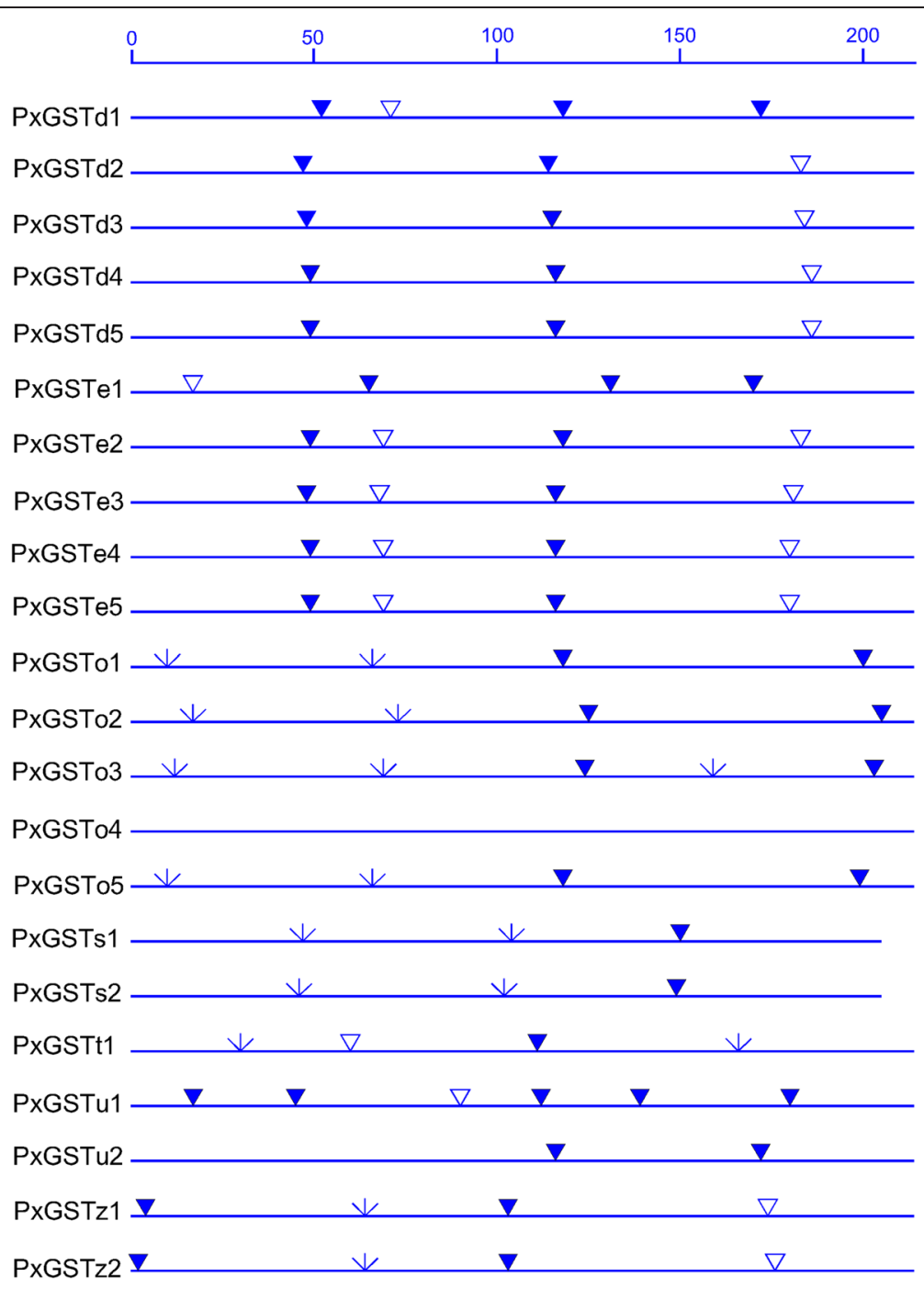

Figure 2 Location of introns of the PxGST genes. Phase 0, 1 and 2 introns are shown by inverted filled triangle, arrow and inverted blank triangle, respectively. Phase 0 for a splice site lying between two codons, phase 1 for a splice site lying one base inside a codon in the $3^{\prime}$ direction, and phase 2 for a splice site lying two bases inside the codon in the $3^{\prime}$ direction.

P. xylostella transcriptomic database, we identified three additional genes. The PxGSTs represented all six subclasses found in other insects [28-30], plus two genes that could not be assigned to any one of the known subclasses, labeled as unclassified. Numbers of GSTs varied greatly across insect species. GSTs were expanded in the Diptera and Coleoptera, with a relatively larger number of total genes than that in the species of Lepidoptera, Hymenoptera and Exopterogota (Table 2). The number of GSTs in P. xylostella was close to that of another lepidopteran species, B. mori. The two insect-specific GST subclasses (Delta and Epsilon) were numerically superior, accounting for $>50 \%$ of the entire cytosolic GSTs in Diptera and Coleoptera and $\sim 50 \%$ in Lepidoptera (Table 2). This indicates that the GSTs in the Delta and
Epsilon subclasses have a greater general trend of duplication than the GSTs in the other four subclasses as previously reported by Friedman [30].

\section{Phylogenetic analysis of the PxGSTs}

The phylogenetic tree illustrated that the seven subclasses were well clustered into their relevant phylogenetic branches (Figure 1). The unclassified subclass diverged from the Delta subclass, suggesting that they may have similar functions. In all the subclasses, the $P$. xylostella GSTs were all clustered into the Lepidopteraspecific branches. Within a specific subclass, the same genes in different species were first clustered into an upper branch within the phylogenetic tree, suggesting that 


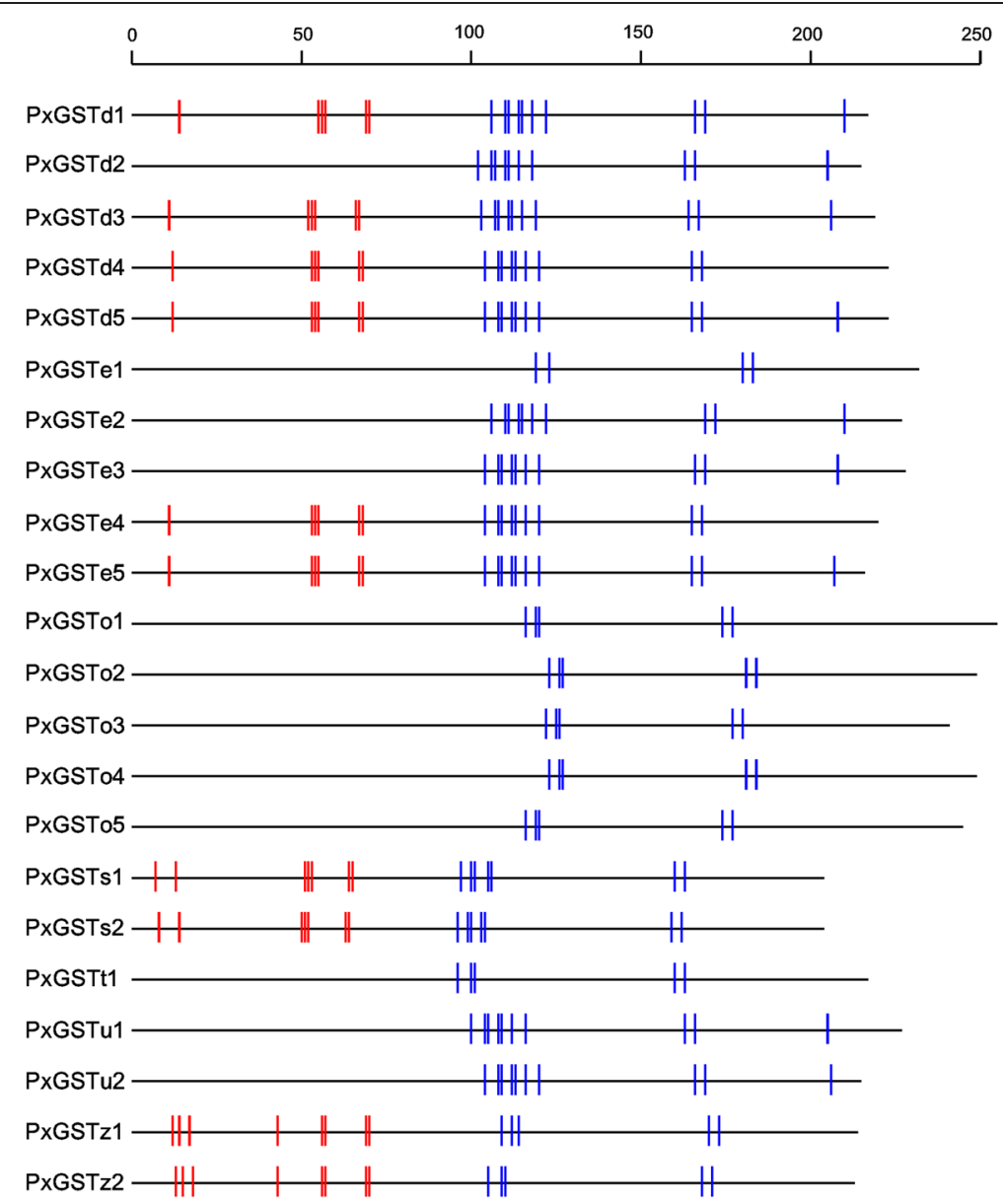

Figure $3 \mathrm{GSH}$ and substrate binding sites of glutathione S-transferase genes. The short vertical lines represent functionally conserved residues of GST genes among insect species. Red vertical lines represent the GSH binding sites of GSTs (G sites) and blue vertical lines represent the substrate binding sites GSTs (H sites).

specific GSTs in different species might have same or similar functions [33,34].

Delta and Epsilon GST subclasses are unique to insects and have been suggested to be implicated in insecticide resistance [10,36,37]. The earlier diverging insects Hymenoptera and Exopterygota do not have Epsilon subclass GSTs. Hymenoptera has a few genes present in the Delta subclass but none for Exopterogota suggesting that these orders may be from an evolutionary older lineage [30]. Our tree suggests that Delta and Epsilon GSTs have diverged more recently from the other subclasses.

The range of amino acid identities in the insectspecific GSTs of $P$. xylostella are fairly variable, ranged from $38.39 \sim 84.75 \%$ in Delta and $23.05 \sim 60.91 \%$ in Epsilon (Additional file 4: Table S1). Except for PxGSTe1, the remaining Epsilon PxGSTs were clustered in a monophyletic clade of Lepidoptera (Figure 1), suggesting a lineage-specific expansion within the Epsilon subclass in lepidopteran order.

\section{Characterization of the PxGST introns}

A total of 80 introns were identified in the PxGSTs. Except for one intronless gene (PxGSTo4), the intron numbers of individual PxGSTs ranged from 2 to 6 (Figure 2) with an average of 3.6. These numbers are similar to those of B. mori GSTs with an average of 3.4 [28] and larger than those of Dipteran (A. gambiae) and Coleopteran (T. castaneum) GSTs with averages of 1.5 and 2.3, respectively [29,38]. The number of GSTs introns has been shown to vary across insect species. It is thought to be associated with the ability to respond to xenobiotics and endogenous compounds [39].

In the PxGSTs, the splice sites of introns were classified into three phases: 0 with 45 introns, 1 with 17 introns, and 2 with 18 introns, according to their positions in the codons. The phase-1 introns were present only in the Omega, Zeta and Sigma subclasses. Most phase-2 introns were found in insect-specific subclasses (Delta and Epsilon) as well as in Zeta subclass. Most of the PxGST introns spliced in a given site tended to be from the 


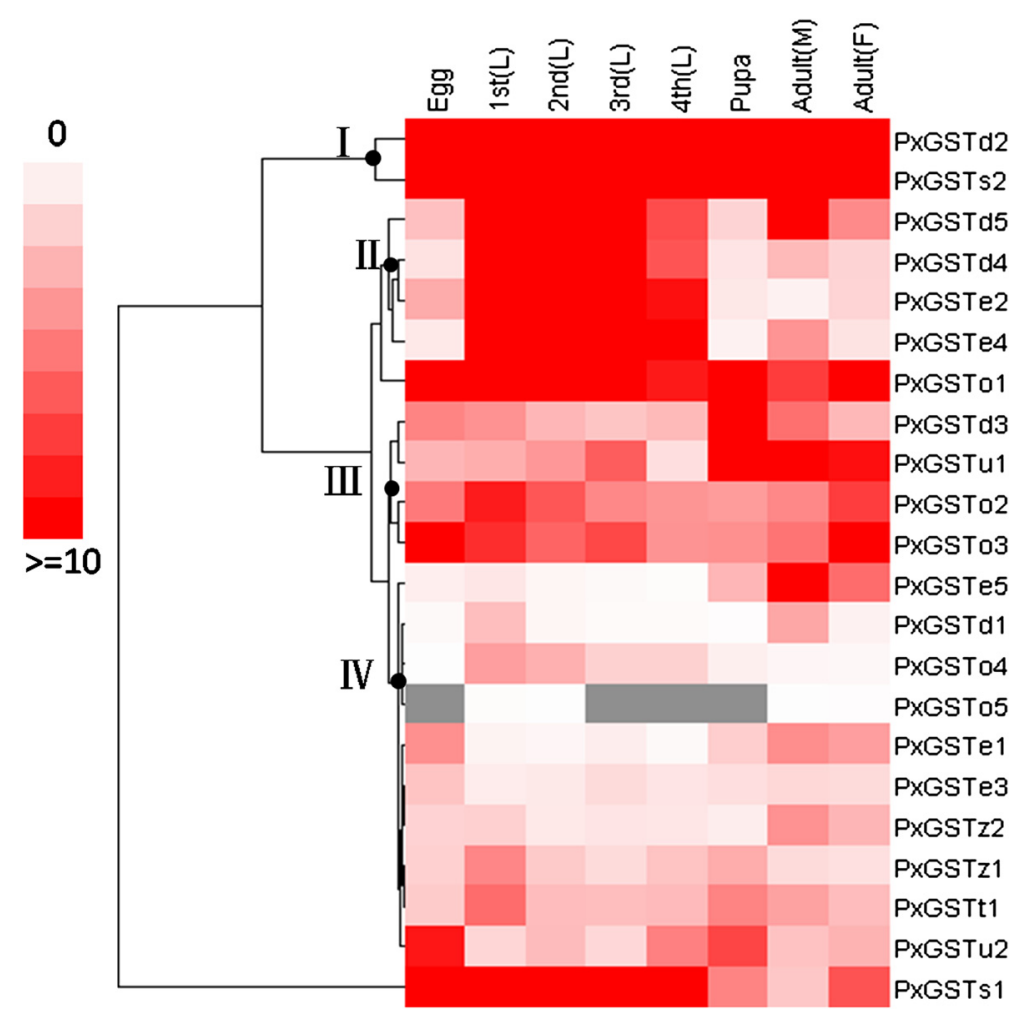

Figure 4 Expression profiling of the PxGSTs at different developmental stages based on RPKM value. 1st (L): first instar larva; 2nd (L): second instar larva; 3rd (L): third instar larva; 4th (L): fourth instar larva; F: female; M: male; Gray denoted missing values. The data were obtained from our unpublished RNA-seq data. The RPKM values are presented in Additional file 7: Table S3. The data have been uploaded to the $P$. xylostella genomic database (DBM-DB: http://iae.fafu.edu.cn/DBM/family/PxGSTs.php).

same phase, suggesting that they might be relatively conserved (Figure 2).

Intron sites are similar across different PxGST subclasses. There are three highly conserved sites of introns within the Delta and Epsilon GST subclasses, except for the PxGSTd1 and PxGSTe1. These were between the $47^{\text {th }}$ and $51^{\text {st }}$, the $114^{\text {th }}$ and $118^{\text {th }}$ and the $180^{\text {th }}$ and $186^{\text {th }}$ amino acids. Most of the PxGSTs tended to have a nearby conserved site of the introns located between the $111^{\text {th }}$ and $125^{\text {th }}$ amino acids belonging to phase 0 . Both of the intron sites and phases were strongly conserved within Sigma and Zeta subclasses (Figure 2), implying that these genes might have similar functions. There appeared to be a correlation between the intron conservation and the phylogenetic cluster within a given PxGST subclass, indicating that gene structure evolution might be involved in the phylogenetic development of a specific subclass.

Despite the conserved nature of intron sites and phases, the lengths were highly variable in the PxGSTs ranging from 28 to $17,644 \mathrm{bp}$ with a larger proportion ranging from 300 to $399 \mathrm{bp}$ (Additional file 5: Figure S1) and an average of $918 \mathrm{bp}$. The shortest intron was PxGSTu1 (28 bp), while the longest were PxGSTo3
(17,644 bp) and PxGSTz2 (13,241 bp). A previous study has shown that long introns were considered to involve more functional elements than short introns and could effectively regulate gene expressions, possibly via the formation of pre-mRNA secondary structures [40]. However, the function of the longest introns in PxGSTo3 and $P x G S T z 2$ needs to be further investigated.

\section{GSH and substrate binding sites in the PxGSTs}

Most of the insect GSTs are composed of a conserved thioredoxin domain containing the GSH binding site (Gsite) and a more variable $\alpha$-helical domain containing the substrate binding site (H-site) [41], and can transfer GSH to a substrate by stabilization of the GSH thiolate [42]. Both G-sites and H-sites among the PxGSTs were analyzed with the NCBI CD-search program, and the results showed that the G-sites appeared fairly conserved while the $\mathrm{H}$-sites were variable among different subclasses (Figure 3, Additional file 6: Table S2). The conserved G-sites indicate their important enzyme functions while the variable $\mathrm{H}$-sites are related to their evolutionary divergence [43]. No G-sites were found for all the genes in Omega GSTs as well as some genes in other subclasses in P. xylostella (Figure 3). 

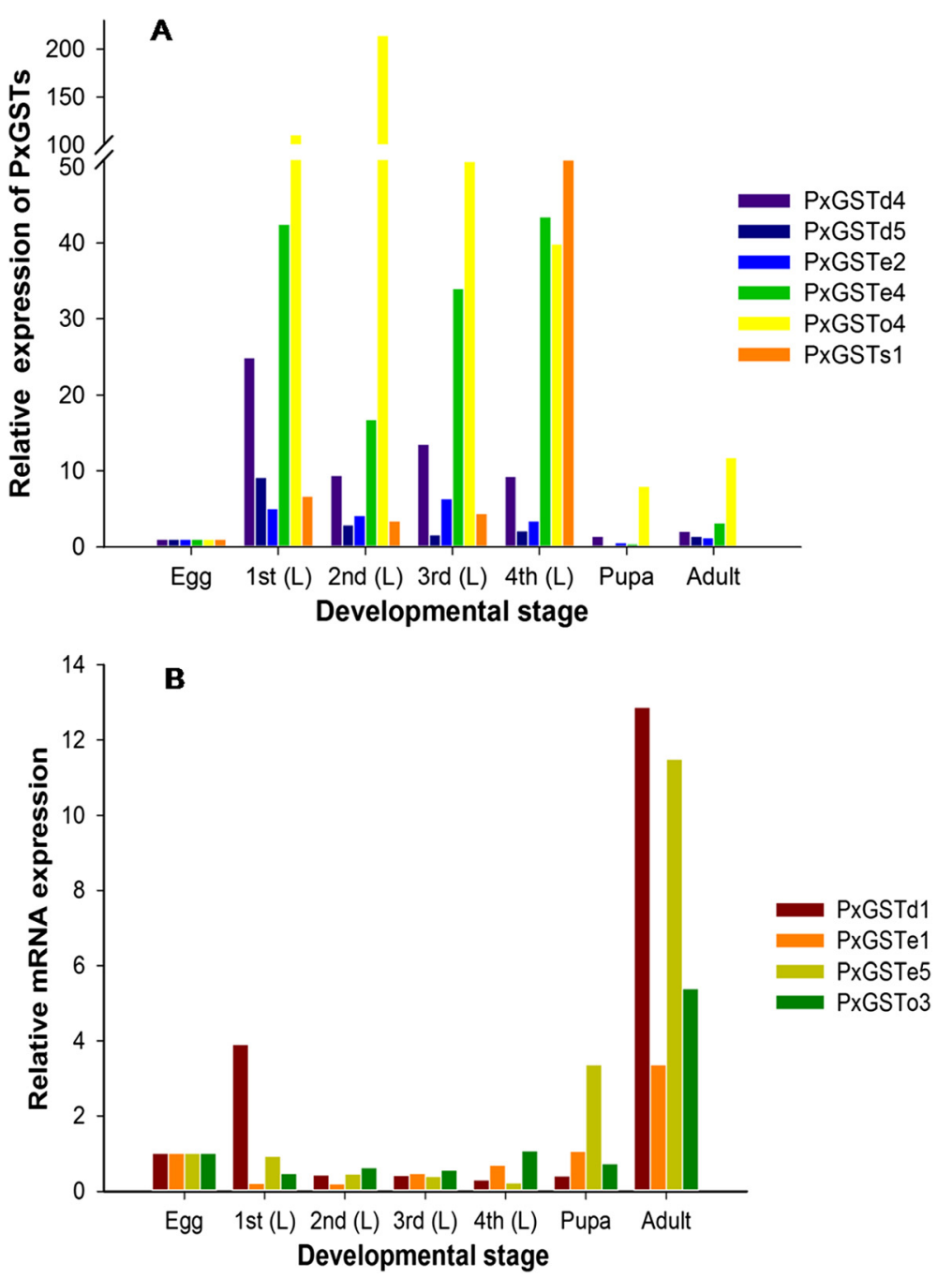

Figure 5 Expression profiling of selected preferentially expressed PxGSTs at larval (A) and adult (B) stages based on qRT-PCR. 1st (L): first instar larva; 2nd (L): second instar larva; 3rd (L): third instar larva; 4th (L): fourth instar larva.

Such GSTs (without G-site) may act as intracellular ligand transporters as documented in Nilaparvata lugens and Anopheles cracens [43,44].

\section{Expression profiling of the PxGSTs \\ Stage-specific expression profiling}

Using our unpublished P. xylostella RNA-seq data, expression patterns of the PxGSTs at different developmental stages of the susceptible strain were characterized (Figure 4, Additional file 7: Table S3). The results showed that all 22 PxGSTs could be expressed at different developmental stages, and exhibited gene-differential and stagespecific patterns. Sixteen genes were found to be consistently expressed throughout different stages, two of which (PxGSTd2 and PxGSTs2, Figure 4, I) tended to be expressed with high levels, four (PxGSTd3, PxGSTu1, PxGSTo2, and PxGSTo3) with moderate levels (Figure 4, III) and ten with low levels (Figure 4, IV). Those highly and moderately expressed genes may function as housekeeping genes with potential roles of protecting cells against endogenous oxidative stress or xenobiotics [28]. Four insect-specific PxGSTs (Figure 4, II) were predominantly and highly expressed at in larval (the main feeding stage), indicating that these genes might play important roles in metabolizing plant secondary metabolites $[45,46]$.

Expression profiling with qRT-PCR confirmed that the 22 PxGSTs genes could express at different developmental stages, but exhibited stage-specific patterns. Six genes were predominantly expressed at the larval stage, exhibiting the same patterns based on RPKM value (Figure 4) and qRT-PCR (Figure 5A), which suggests that they might be associated with detoxification of plant defense compounds and insecticides $[34,46]$. The insect-specific 


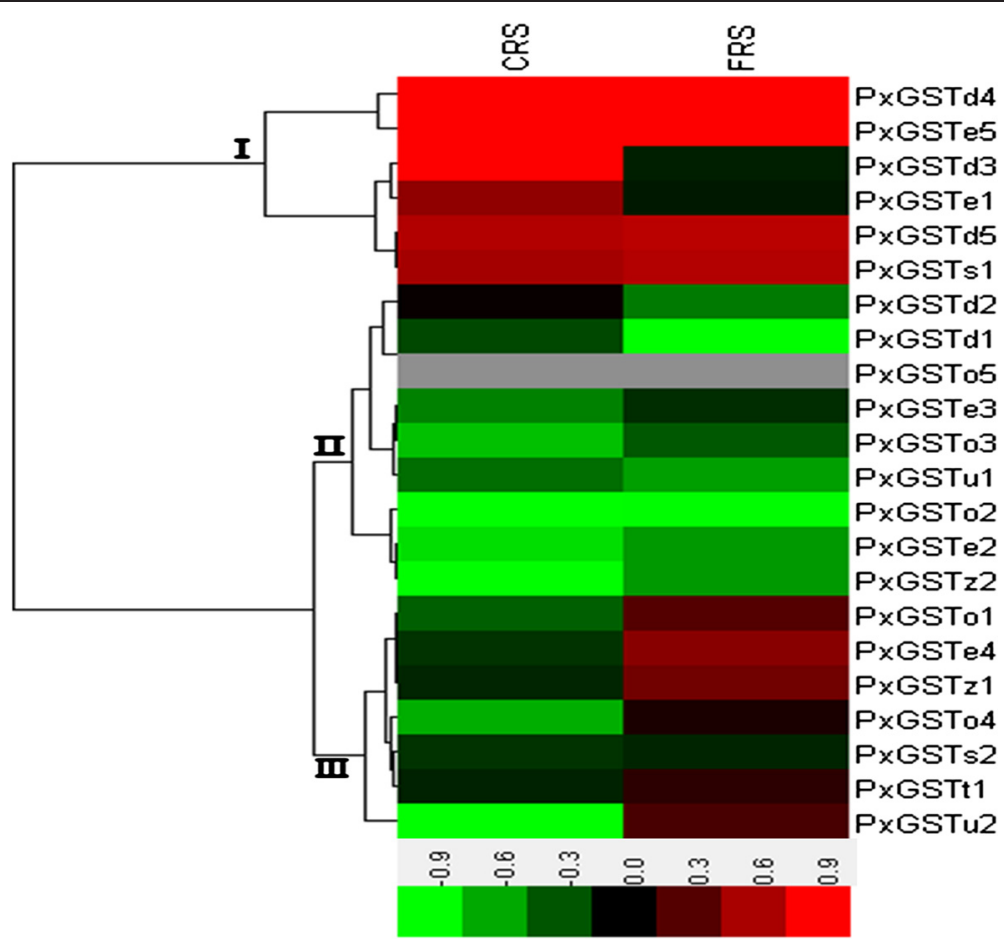

Figure 6 Differential expressions of the PxGSTs in different resistant strains based on RPKM value. CRS: chlorpyrifos resistant strain; FRS: fipronil resistant strain. Differential expressions are illustrated by different colors compared to the expression in susceptible strain (SS), with the red representing up-regulated, the green down-regulated and the black no difference with SS. The gray denotes missing values. The data were obtained from our published transcriptome data. The RPKM values are presented in Additional file 8: Table S4. The data have been uploaded to the P. xylostella genomic database (DBM-DB: http://iae.fafu.edu.cn/DBM/family/PxGSTs.php).

Delta and Epsilon GSTs showed high expression in $P$. xylostella (Figure 5A), while most of these GSTs had little or no expression in the main detoxification organ (fat body) of domesticated $B$. mori with little exposure to insecticides for thousands years [28], suggesting that these genes are associated with the evolution of insecticide resistance as proposed in previous reports [34,45]. PxGSTd1, PxGSTe1, PxGSTe5 and PxGSTo3 were highly expressed in the P. xylostella adults (Figure 5B), suggesting that these genes may be involved in odorant processing and/or xenobiotic metabolism [22]. Most of the PxGSTs exhibited low gene expression at the egg stage (Figure 5). Such diversified expression patterns of the PxGSTs imply that GSTs may have multiple functions in P. xylostella, as documented in other insects $[34,47,48]$.

\section{Strain- and tissue-specific expression profiling}

Based on the DBM transcriptome, all of the PxGSTs were either up- or down-regulated in the insecticide resistant strains when compared to the susceptible strain (SS) (Figure 6, Additional file 8: Table S4). Seven PxGSTs were up-regulated in both of the chlorpyrifos- and fipronilresistant strains (CRS and FRS) (Figure 6, group I). They are mostly insect specific GSTs (Delta and Epsilon) with the potential function of detoxification. However, seven
PxGSTs were down-regulated in CRS and FRS (Figure 6, group II). Most of the rest were up-regulated in FRS, but down- regulated in CRS, possibly reflecting different mechanisms of detoxification between the two strains.

The qRT-PCR-based analysis showed that four of the PxGSTs (PxGSTd3, PxGSTd4, PxGSTo2 and PxGSTo5) showed significantly greater expression in both CRS and FRS. PxGSTd5, PxGSTs1, PxGSTs 2 and PxGSTz2 in FRS and PxGSTo1 and PxGSTo4 in CRS also had greater gene expressions than in SS. Interestingly, PxGSTe4 and PxGSTt1 exhibited higher expressions in FRS but lower expressions in CRS when compared to the SS (Figure 7). Expression of the other 10 PxGSTs were not significantly different among strains. The qRT-PCR-based analysis could not confirm the transcriptome-based expression profiling patterns of all the PxGSTs, which might result from different sampling times when the resistant DBMs (FRS and CRS) were collected for transcriptome sequencing in December 2009, and collected three years later for $\mathrm{qRT}$-PCR. The diversified patterns of strain-specific expressions suggest that the PxGSTs might involve a functionally complex system in response to detoxifying different classes of insecticides [34,49,50].

Twelve PxGSTs with significantly high expression in resistant strains were further analyzed using qRT-PCR in 


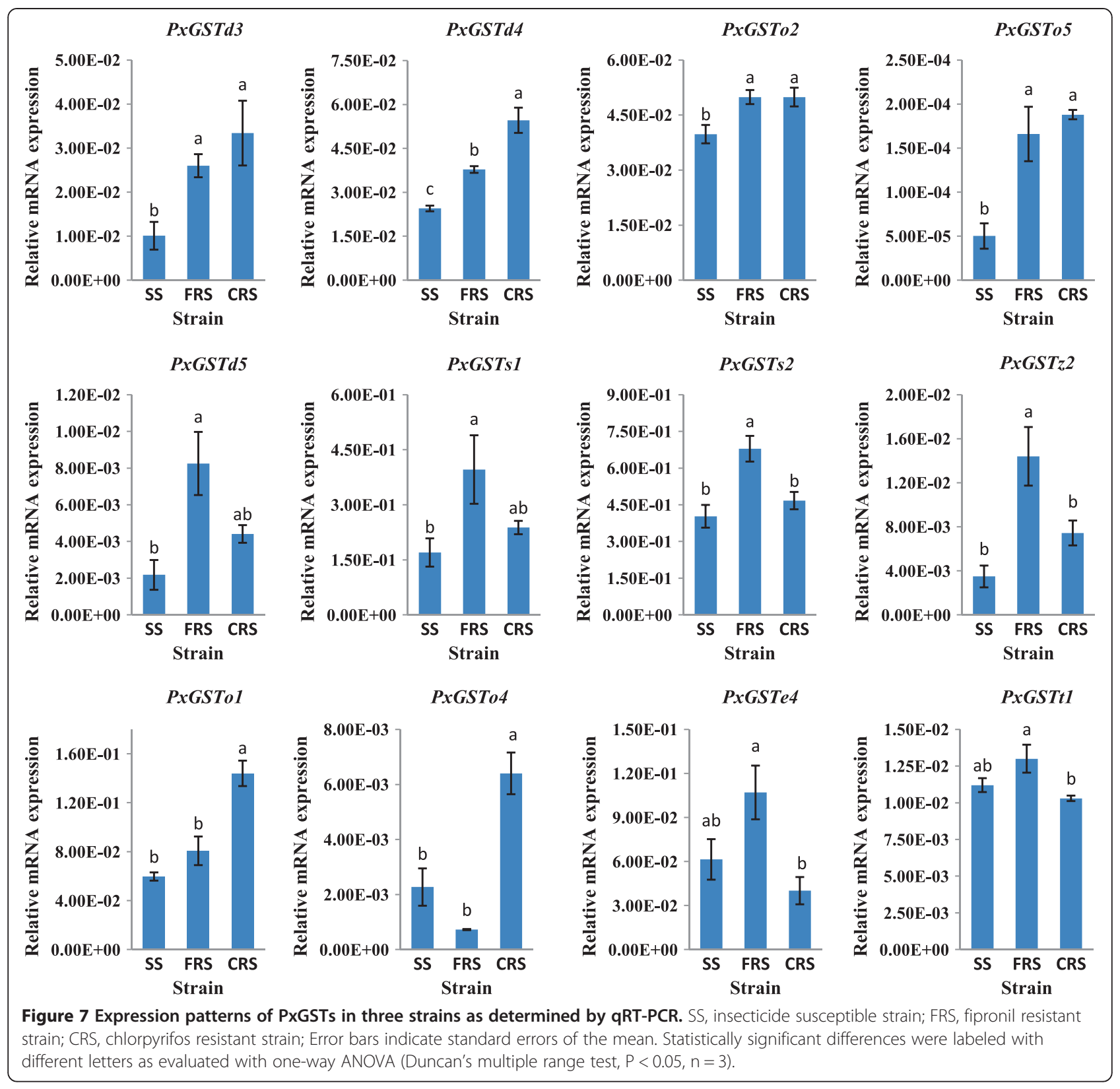

different tissues of fipronil-resistant strain. The results showed that 6 genes (PxGSTd3, PxGSTd5, PxGSTs2, PxGSTz2, PxGSTo1 and PxGSTo4) were significantly more highly expressed in Malpighian tubules, 4 (PxGSTd4, PxGSTo2, PxGSTe4 and PxGSTt1) both in Malpighian tubules and midguts and 1 (PxGSTs1) in epidermis, except for PxGSTo5 with no significant different expression in the various tissues (Figure 8). These results further validate the association of the PxGSTs with insecticide detoxification because these tissues were documented to play important roles in digestion and metabolism of xenobiotics in insects [51,52].

\section{Conclusions}

To date, this is the most comprehensive study on genome-wide identification, characterization and expression profile of the GSTs in P. xylostella. Twenty-two GSTs were found in P. xylostella, which is similar in number to another lepidopteran species, B. mori. Variable features and different expression patterns of the genes reveal that the P. xylostella GSTs are evolutionary and functionally diversified, and may be involved in the evolution of adaptive capacity in response to environmental variation. Because GST enzymes are considered to be important in insecticide resistance, many of these 


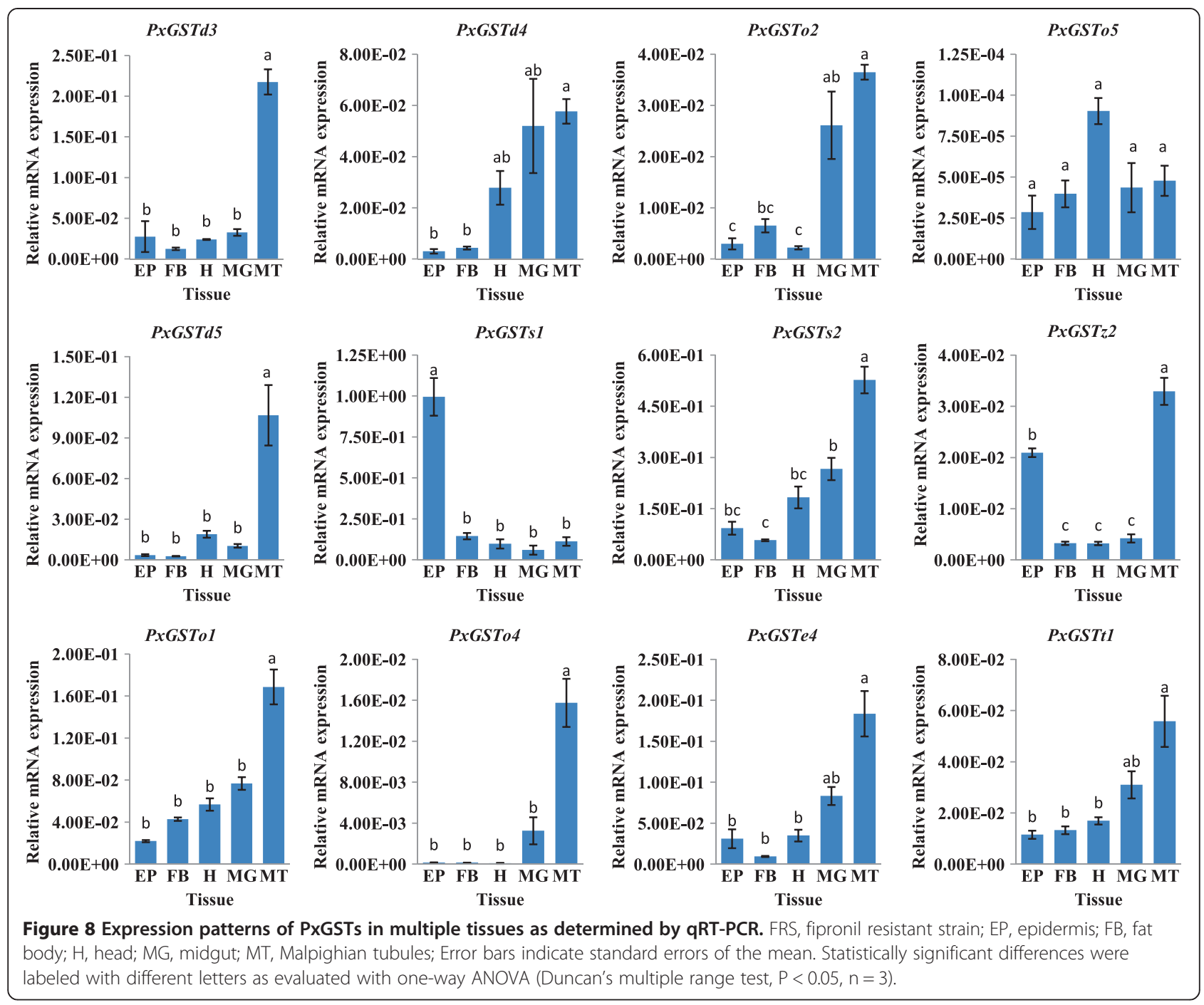

newly identified genes are potential candidates for inhibiting the pathway of insecticide resistance and targeting lepidopteran-selective insecticides. Thus, further functional research on the PxGSTs is essential to identify the key genes and their roles in xenobiotic detoxification of insects, and understand the mechanisms underlying the insecticide resistance.

\section{Methods}

\section{Experimental DBM strains}

The experimental population of P. xylostella was derived from a susceptible strain (SS) that was collected from a vegetable field of Fuzhou $\left(26.08^{\circ} \mathrm{N}, 119.28^{\circ} \mathrm{E}\right)$ in 2004 and used for genome sequencing [23]. Since then this initial population was reared on potted radish seedlings (Raphanus sativus L.) at $25 \pm 1{ }^{\circ} \mathrm{C}, 65 \pm 5 \%$ $\mathrm{RH}$ and $\mathrm{L}: \mathrm{D}=16: 8 \mathrm{~h}$ in a separate greenhouse without exposure to insecticides over the past ten years. Two insecticide resistant strains (chlorpyrifos- and fipronil- resistant strains (CRS and FRS)) were selected from this susceptible strain, and detailed in DBM transcriptome [26].

\section{Identification of $P$. xylostella GST genes}

To identify putative GST genes from the DBM genome database [23,27], the GST protein sequences of $D$. melanogaster, C. quinquefasciatus, A. aegypti, A. gambiae, T. castaneum, A. mellifera, $N$. vitripennis, $A$. pisum, $P$. humanus, $B$. $m o r i$ and other lepidopteran insects were downloaded from their genome databases [53-57] and/or GenBank (http://www.ncbi.nlm.nih.gov/) and Uniprot (http://www. uniprot.org/). These insect GST protein sequences were used as queries to perform local TBLASTN searches against the DBM genome database. The putative genomic sequences were retrieved, and then predicted using Fgenesh + (http://www.softberry.com/). The DBM GST protein sequences were confirmed using online BLASTP in NCBI. 


\section{Phylogenetic analysis}

The GSTs of A. aegypti (Aa), A. gambiae (Ag), D. melanogaster (Dm), A. mellifera (Am), N. vitripennis (Nv), Papilio polytes $(P p)$, Danaus plexippus $(D p)$, B. mori $(B m)$ and $P$. xylostella $(P x)$ were used for the phylogenetic analysis. Putative amino acid sequences of the GSTs were aligned using Clustal X2.0 [58], and then gaps and missing data were manually trimmed. A phylogenetic tree was constructed with the neighbor-joining method [59] using MEGA 5.10 [35]. Bootstrap analysis with 1,000 replicates was used to evaluate the significance of the nodes. Poisson correction amino acid model and pairwise deletion of gaps were selected for the tree reconstruction.

\section{RNA extraction and CDNA synthesis}

DBM eggs, $1^{\text {st }}$ - to $4^{\text {th }}$-instar larvae, pupae and adults from susceptible and resistant strains were frozen in liquid nitrogen. Total RNA was extracted using the RNAiso Plus (Takara, Code: D9108A, Japan). The $4^{\text {th }}$-instar larvae were surface sterilized in $75 \%$ ethanol, then dipped in DNAase and RNAse free water and dissected. Tissues (head, midgut, Malpighian tubules, fatbody and epidermis) from resistant strains were briefly immersed in RNAlater ${ }^{\text {Tu }}$ RNA Stabilization Reagent (QIAGEN, Code: 76104, Germany) then stored at $4^{\circ} \mathrm{C}$. Total RNA was extracted with the RNeasy Plus Micro Kit (QIAGEN, Code: 74034, Germany) and RNA concentration was determined using a spectrophotometer (Nanodrop 2000: Thermo, USA).

The cDNA template for PCR was synthesized with $1 \mu \mathrm{g}$ of total RNA using PrimeScript ${ }^{\circ} \mathrm{RT}$ reagent Kit with gDNA Eraser (Perfect Real Time) (Takara, Code: DRR047A, Japan).

\section{Validation of gene expression by qRT-PCR}

The qRT-PCR primers used in the validation of gene expression were identified based on the encoding sequences of the DBM GSTs (Additional file 9: Table S5). DBM ribosomal protein L8 (RPL8) was used as reference gene for different strains and tissues, and DBM ribosomal protein S4 (RPS4) for different stages/instars. The assays were run in triplicate in CFX96 Touch $^{\text {Tw }}$ RealTime PCR Detection Systems (Bio-Rad, USA). PCR amplification was performed in a total reaction volume of $20 \mu \mathrm{L}$ reaction mixture, containing $20 \mathrm{ng}$ cDNA, $10 \mu \mathrm{L} 2 \times$ SYBR $^{\circ}$ Premix Ex Taq ${ }^{\text {Tu }}$ (Takara, DRR420A, Japan), $0.2 \mu \mathrm{M}$ of each primer. PCR was conducted with standard thermal cycle conditions using the two-step qRT-PCR method: an initial denaturation at $95^{\circ} \mathrm{C}$ for $30 \mathrm{~s}$ followed by 40 cycles of $3 \mathrm{~s}$ at $95^{\circ} \mathrm{C}$ and $30 \mathrm{~s}$ at $60^{\circ} \mathrm{C}$. Specificity of the PCR products was assessed by melting curve analysis for all samples. For each treatment (tissues, strains and developmental stages), there were three biological replicates.

\section{Statistical analysis}

The $2^{-\Delta C t}$ method was used to analyze the qRT-PCRbased expression patterns. One-way ANOVA, using PASW Statistics 18, followed by a Duncan's multiple range test was used to evaluate significant differences among patterns. The results were presented by mean \pm standard deviation of the relative mRNA expressions.

\section{Availability of supporting data}

The nucleic acid sequences and protein sequences have been deposited in the published DBM genomic database (DBM-DB: http://iae.fafu.edu.cn/DBM/family/PxGSTs.php). Other supporting data are presented in Additional file 7: Tables S3 and Additional file 8: Table S4, and also deposited in the same database.

\section{Additional files}

Additional file 1: Coding sequences of PxGSTs.

Additional file 2: Amino acid sequences of PxGSTs.

Additional file 3: Genomic DNA sequences of PxGSTs.

Additional file 4: Table S1. Subclass-based matrix of the amino acid identity among different PXGSTs.

Additional file 5: Figure S1. Numerical distribution of intron length in the PXGSTS.

Additional file 6: Table S2. GSH and substrate binding sites of the PXGSTS.

Additional file 7: Table S3. RPKM of the PXGSTs at different developmental stages obtained from RNA-seq data.

Additional file 8: Table S4. RPKM of PXGST genes in different strains obtained from transcriptome data.

Additional file 9: Table S5. Primer sequences of $q R T-P C R$

\section{Competing interests}

The authors declare that they have no competing interests.

\section{Authors' contributions}

YY and MX designed and performed experiments, analyzed data and co-wrote the paper. NR, XC, JL, XM and MZ helped in the diamondback moth materials collection, total RNA extraction and qRT-PCR. MY, LV and GMG supervised the analyses and critically revised the manuscript. All authors have read and approved the final manuscript.

\section{Acknowledgements}

We are grateful to Liying Yu for her advice on analysis of gene family and phylogenetic analysis, and to Drs. Simon W. Baxter and Mark S. Goettel for their comments on our manuscript at early stage. We thank Yimin Zhuo, Jinyang Cai, Qiuye Qiu, Wenxiu Xie, Xianbin Huang and Xiaohui Huang for their technical assistance in rearing diamondback moth and preparing samples. The work was supported by National Natural Science Foundation of China (No. 31320103922 and No. 31230061) and National Key Project of Fundamental Scientific Research ("973" Programs, No. 2011CB100404) in China. LV is supported by the Minjiang Scholar Program in Fujian Province (PRC), and GMG by the National Thousand Talents Program in China.

\section{Author details}

${ }^{1}$ Institute of Applied Ecology and Research Centre for Biodiversity and Eco-Safety, Fujian Agriculture and Forestry University, Fuzhou 350002, China. ${ }^{2}$ College of Life Science, Fujian Agriculture and Forestry University, Fuzhou 350002, China. ${ }^{3}$ Key Laboratory of Integrated Pest Management of Fujian and Taiwan, China Ministry of Agriculture, Fuzhou 350002, China. ${ }^{4}$ Department of Biological Sciences, Brock University, 500 Glenridge Avenue, St. Catharines, 
ON L2S 3A1, Canada. ${ }^{5}$ EH Graham Centre, Charles Sturt University, Orange, NSW 2800, Australia.

\section{Received: 12 September 2014 Accepted: 12 February 2015 Published online: 05 March 2015}

\section{References}

1. Furlong MJ, Wright DJ, Dosdall LM. Diamondback moth ecology and management: problems, progress, and prospects. Annu Rev Entomol. 2013;58:517-41.

2. Georghiou GP. Overview of insecticide resistance. In: Managing resistance to agrochemicals. vol. 421. Washington, DC: American Chemical Society; 1990. p. 18-41.

3. Sonoda S. Molecular analysis of pyrethroid resistance conferred by target insensitivity and increased metabolic detoxification in Plutella xylostella. Pest Manage Sci. 2010;66(5):572-5.

4. Kim Jl, Joo YR, Kwon M, Kim GH, Lee SH. Mutation in acel associated with an insecticide resistant population of Plutella xylostella. J Asia-Pacif Entomol. 2012;15(3):401-7.

5. Dukre AS, Moharil MP, Ghodki BS, Rao NGV. Role of glutathione Stransferase in imparting resistance to pyrethroids in Plutella xylostella(L.). Int J Integr Bio. 2009;6(1):17-21.

6. Ranson H, Hemingway J. 5.11 - Glutathione Transferases. In: Gilbert LI, editor. Compr Mol Insect Sci. Amsterdam: Elsevier; 2005. p. 383-402.

7. Enayati AA, Ranson H, Hemingway J. Insect glutathione transferases and insecticide resistance. Insect Mol Biol. 2005;14(1):3-8.

8. Clark AG. The comparative enzymology of the glutathione S-transferases from non-vertebrate organisms. Comp Biochem Physiol B: Comp Biochem. 1989;92(3):419-46

9. Fournier D, Bride JM, Poirie M, Berge JB, Plapp Jr FW. Insect glutathione S-transferases. Biochemical characteristics of the major forms from houseflies susceptible and resistant to insecticides. J Biol Chem. 1992;267(3):1840-5.

10. Ranson H, Rossiter L, Ortelli F, Jensen B, Wang X, Roth CW, et al. Identification of a novel class of insect glutathione S-transferases involved in resistance to DDT in the malaria vector Anopheles gambiae. Biochem J. 2001:359(Pt 2):295-304

11. Enayati AA, Vontas JG, Small GJ, McCarroll L, Hemingway J. Quantification of pyrethroid insecticides from treated bednets using a mosquito recombinant glutathione S-transferase. Med Vet Entomol. 2001;15(1):58-63.

12. Chelvanayagama G, Parker MW, Board PG. Fly fishing for GSTs: A unified nomenclature for mammalian and insect glutathione transferases. Chem-Biol Interact. 2001;133:256-60.

13. Low WY, Ng HL, Morton CJ, Parker MW, Batterham P, Robin C. Molecular evolution of glutathione S-transferases in the genus Drosophila. Genetics. 2007;177(3):1363-75.

14. Sawicki R, Singh SP, Mondal AK, Benes H, Zimniak P. Cloning, expression and biochemical characterization of one Epsilon-class (GST-3) and ten Delta-class (GST-1) glutathione S-transferases from Drosophila melanogaster, and identification of additional nine members of the Epsilon class. Biochem J. 2003:370(Pt 2):661-9.

15. Ketterman AJ, Saisawang C, Wongsantichon J. Insect glutathione transferases. Drug Metab Rev. 2011:43(2):253-65.

16. Abel EL, Bammler TK, Eaton DL. Biotransformation of methyl parathion by glutathione S-transferases. Toxicol Sci. 2004;79(2):224-32.

17. Huang HS, Hu NT, Yao YE, Wu CY, Chiang SW, Sun CN. Molecular cloning and heterologous expression of a glutathione S-transferase involved in insecticide resistance from the diamondback moth. Plutella xylostella Insect Biochem Mol Bio. 1998;28(9):651-8.

18. Wei SH, Clark AG, Syvanen M. Identification and cloning of a key insecticidemetabolizing glutathione S-transferase (MdGST-6A) from a hype insecticide-resistant strain of the housefly Musca domestica. Insect Biochem Mol Bio. 2001;31(12):1145-53.

19. Hemingway J, Miyamoto J, Herath PRJ. A possible novel link between organophosphorus and DDT insecticide resistance genes in Anopheles: Supporting evidence from fenitrothion metabolism studies. Pestic Biochem Physiol. 1991;39(1):49-56

20. Yamamoto K, Shigeoka Y, Aso Y, Banno Y, Kimura M, Nakashima T. Molecular and biochemical characterization of a Zeta-class glutathione S-transferase of the silkmoth. Pestic Biochem Physiol. 2009;94(1):30-5.
21. Yamamoto K, Nagaoka S, Banno Y, Aso Y. Biochemical properties of an omega-class glutathione S-transferase of the silkmoth, Bombyx mori. Comp Biochem Physiol C: Toxicol Pharmacol. 2009;149(4):461-7.

22. Rogers ME, Jani MK, Vogt RG. An olfactory-specific glutathione-S-transferase in the sphinx moth Manduca sexta. J Exp Biol. 1999;202(Pt 12):1625-37.

23. You M, Yue Z, He W, Yang X, Yang G, Xie M, et al. A heterozygous moth genome provides insights into herbivory and detoxification. Nat Genet. 2013;45(2):220-5.

24. Chen X, Zhang YL. Identification and characterisation of multiple glutathione S-transferase genes from the diamondback moth, Plutella xylostella. Pest Manag Sci. 2014

25. Jouraku A, Yamamoto K, Kuwazaki S, Urio M, Suetsugu Y, Narukawa J, et al. KONAGAbase: a genomic and transcriptomic database for the diamondback moth, Plutella xylostella. BMC Genomics. 2013;14:464.

26. He W, You M, Vasseur L, Yang G, Xie M, Cui K, et al. Developmental and insecticide-resistant insights from the de novo assembled transcriptome of the diamondback moth. Plutella xylostella Genomics. 2012;99(3):169-77.

27. Tang W, Yu L, He W, Yang G, Ke F, Baxter SW, et al. DBM-DB: the diamondback moth genome database. Database (Oxford). 2014;2014:bat087.

28. Yu Q, Lu C, Li B, Fang S, Zuo W, Dai F, et al. Identification, genomic organization and expression pattern of glutathione S-transferase in the silkworm, Bombyx mori. Insect Biochem Mol Bio. 2008;38(12):1158-64.

29. Ding Y, Ortelli F, Rossiter LC, Hemingway J, Ranson H. The Anopheles gambiae glutathione transferase supergene family: annotation, phylogeny and expression profiles. BMC Genomics. 2003;4(1):35

30. Friedman R. Genomic organization of the glutathione S-transferase family in insects. Mol Phylogen Evol. 2011;61(3):924-32.

31. Oakeshott JG, Johnson RM, Berenbaum MR, Ranson H, Cristino AS, Claudianos C. Metabolic enzymes associated with xenobiotic and chemosensory responses in Nasonia vitripennis. Insect Mol Biol. 2010;19:147-63.

32. Nair PMG, Choi J. Identification, characterization and expression profiles of Chironomus riparius glutathione S-transferase (GST) genes in response to cadmium and silver nanoparticles exposure. Aquat Toxicol. 2011;101(3-4):550-60.

33. Deng H, Huang Y, Feng Q, Zheng S. Two epsilon glutathione S-transferase cDNAs from the common cutworm, Spodoptera litura: Characterization and developmental and induced expression by insecticides. J Insect Physiol. 2009:55(12):1174-83.

34. Lumjuan N, Rajatileka S, Changsom D, Wicheer J, Leelapat P, Prapanthadara $\mathrm{L}-\mathrm{a}$, et al. The role of the Aedes aegypti Epsilon glutathione transferases in conferring resistance to DDT and pyrethroid insecticides. Insect Biochem Mol Bio. 2011:41(3):203-9.

35. Tamura K, Peterson D, Peterson N, Stecher G, Nei M, Kumar S. MEGA5: molecular evolutionary genetics analysis using maximum likelihood, evolutionary distance, and maximum parsimony methods. Mol Biol Evol. 2011;28(10):2731-9.

36. Vontas JG, Small GJ, Nikou DC, Ranson H, Hemingway J. Purification, molecular cloning and heterologous expression of a glutathione S-transferase involved in insecticide resistance from the rice brown planthopper, Nilaparvata lugens. Biochem J. 2002;362(Pt 2):329-37.

37. Wang JY, McCommas S, Syvanen M. Molecular cloning of a glutathione S-transferase overproduced in an insecticide-resistant strain of the housefly (Musca domestica). Mol Gen Genet. 1991:227(2):260-6.

38. Shi H, Pei L, Gu S, Zhu S, Wang Y, Zhang Y, et al. Glutathione S-transferase (GST) genes in the red flour beetle, Tribolium castaneum, and comparative analysis with five additional insects. Genomics. 2012;100(5):327-35.

39. Savard J, Tautz D, Richards S, Weinstock GM, Gibbs RA, Werren JH, et al. Phylogenomic analysis reveals bees and wasps (Hymenoptera) at the base of the radiation of Holometabolous insects. Genome Res. 2006:16(11):1334-8.

40. Haddrill PR, Charlesworth B, Halligan DL, Andolfatto P. Patterns of intron sequence evolution in Drosophila are dependent upon length and GC content. Genome Biol. 2005;6(8):R67

41. Atkinson HJ, Babbitt PC. Glutathione transferases are structural and functiona outliers in the thioredoxin fold. Biochemistry. 2009;48(46):11108-16.

42. Armstrong RN. Structure, catalytic mechanism, and evolution of the glutathione transferases. Chem Res Toxicol. 1997;10(1):2-18.

43. Zhou WW, Liang QM, Xu Y, Gurr GM, Bao YY, Zhou XP, et al. Genomic insights into the gutathione S-transferase gene family of two rice planthoppers, Nilaparvata lugens (Stal) and Sogatella furcifera (Horvath) (Hemiptera: Delphacidae). PLoS One. 2013;8(2):e56604. 
44. Wongtrakul J, Pongjaroenkit S, Leelapat P, Nachaiwieng W, Prapanthadara LA, Ketterman AJ. Expression and characterization of three new glutathione transferases, an epsilon (AcGSTE2-2), omega (AcGSTO1-1), and theta (AcGST1-1) from Anopheles cracens (Diptera: Culicidae), a major Thai malaria vector. J Med Entomol. 2010;47(2):162-71.

45. Li X, Schuler MA, Berenbaum MR. Molecular mechanisms of metabolic resistance to synthetic and natural xenobiotics. Annu Rev Entomol. 2007;52:231-53.

46. Sun $X Q$, Zhang MX, Yu JY, Jin Y, Ling B, Du JP, et al. Glutathione Stransferase of brown planthoppers (Nilaparvata lugens) is essential for their adaptation to gramine-containing host plants. PLoS One. 2013;8(5):e64026.

47. Zhang Y, Yan H, Lu W, Li Y, Guo X, Xu B. A novel Omega-class glutathione S-transferase gene in Apis cerana cerana: molecular characterisation of GSTO2 and its protective effects in oxidative stress. Cell Stress Chaperones. 2013;18(4):503-16.

48. Lumjuan N, McCarroll L, Prapanthadara LA, Hemingway J, Ranson H. Elevated activity of an Epsilon class glutathione transferase confers DDT resistance in the dengue vector, Aedes aegypti. Insect Biochem Mol Bio. 2005;35(8):861-71.

49. Yamamoto $K$, Ichinose H, Aso Y, Banno Y, Kimura M, Nakashima T. Molecular characterization of an insecticide-induced novel glutathione transferase in silkworm. Biochim Biophys Acta. 2011;1810(4):420-6.

50. Samra Al, Kamita SG, Yao H-W, Cornel AJ, Hammock BD. Cloning and characterization of two glutathione S-transferases from pyrethroid-resistant Culex pipiens. Pest Manage Sci. 2012;68(5):764-72.

51. Dow JA. Insights into the Malpighian tubule from functional genomics. J Exp Biol. 2009;212(Pt 3):435-45.

52. Yu QY, Lu C, Li WL, Xiang ZH, Zhang Z. Annotation and expression of carboxylesterases in the silkworm, Bombyx mori. BMC Genomics. 2009; 10:553.

53. Megy K, Emrich SJ, Lawson D, Campbell D, Dialynas E, Hughes DS, et al. VectorBase: improvements to a bioinformatics resource for invertebrate vector genomics. Nucleic Acids Res. 2012;40(Database issue):D729-34.

54. Marygold SJ, Leyland PC, Seal RL, Goodman JL, Thurmond J, Strelets VB, et al. FlyBase: improvements to the bibliography. Nucleic Acids Res. 2013;41(D1):D751-7.

55. Legeai F, Shigenobu S, Gauthier JP, Colbourne J, Rispe C, Collin O, et al. AphidBase: a centralized bioinformatic resource for annotation of the pea aphid genome. Insect Mol Biol. 2010;19 Suppl 2:5-12.

56. Kim HS, Murphy T, Xia J, Caragea D, Park Y, Beeman RW, et al. BeetleBase in 2010: revisions to provide comprehensive genomic information for Tribolium castaneum. Nucleic Acids Res. 2010;38(Database issue):D437-42

57. Munoz-Torres MC, Reese JT, Childers CP, Bennett AK, Sundaram JP, Childs $\mathrm{KL}$, et al. Hymenoptera Genome Database: integrated community resources for insect species of the order Hymenoptera. Nucleic Acids Res. 2011;39(Database issue):D658-62.

58. Larkin MA, Blackshields G, Brown NP, Chenna R, McGettigan PA, McWilliam $\mathrm{H}$, et al. Clustal W and Clustal X version 2.0. Bioinformatics. 2007;23(21):2947-8.

59. Saitou N, Nei M. The neighbor-joining method: a new method for reconstructing phylogenetic trees. Mol Biol Evol. 1987;4(4):406-25.

\section{Submit your next manuscript to BioMed Central and take full advantage of:}

- Convenient online submission

- Thorough peer review

- No space constraints or color figure charges

- Immediate publication on acceptance

- Inclusion in PubMed, CAS, Scopus and Google Scholar

- Research which is freely available for redistribution 